\title{
Supporting the Management of Patients with Heart Failure within Asia-Pacific, Middle East, and African Countries: A Toolbox for Healthcare Providers
}

\author{
Samer Kabbani ${ }^{a}$ Waleed Al Habeeb ${ }^{b}$ Houng-Bang Liew ${ }^{c}$ Jagdish C. Mohan ${ }^{d}$ \\ Elijah Ogola ${ }^{e}{\text { David } \operatorname{Sim}^{f} \text { Nqoba Tsabedze }}^{g}$ \\ ${ }^{a}$ Division of Cardiology, Department of Internal Medicine, Rafik Hariri University Hospital, Beirut, Lebanon; \\ ${ }^{b}$ Department of Cardiac Sciences, College of Medicine, King Saud University, Riyadh, Saudi Arabia; 'Sabah Heart \\ Centre, Queen Elizabeth Hospital, Kota Kinabalu, Malaysia; ${ }^{d}$ Institute of Heart and Vascular Disorders, Jaipur Golden \\ Hospital, Rohini, Delhi, India; 'Department of Clinical Medicine and Therapeutics, University of Nairobi, Nairobi, \\ Kenya; ${ }^{f}$ Department of Cardiology, National Heart Centre Singapore, Singapore, Singapore; ${ }^{9}$ Division of Cardiology, \\ Department of Internal Medicine, Faculty of Health Sciences, University of the Witwatersrand, Charlotte Maxeke \\ Johannesburg Academic Hospital, Gauteng, South Africa
}

\section{Keywords \\ Heart failure management toolbox · Asia-Pacific · Middle \\ East and Africa - Acute heart failure - Chronic heart \\ failure with reduced ejection fraction - Multidisciplinary \\ management · Emergency setting · Hospital setting · \\ Outpatient setting · Practical resources}

\begin{abstract}
Objectives: The management of heart failure (HF) is most effective when established treatment guidelines and recommendations are followed. We aimed to develop a "Toolbox" of resources to facilitate the care of patients with acute HF and chronic HF with reduced ejection fraction delivered by healthcare professionals across Asia-Pacific, the Middle East and Africa (henceforth referred to as the "Region"). Methods: We convened a group of cardiologists from across the Region to develop a set of checklists, algorithms, and other practical resources. These resources are based on our experiences, current evidence, and international guidelines. $\boldsymbol{R} \boldsymbol{e}$ sults: The HF Toolbox comprises three simplified sets of re-
\end{abstract}

sources for use in the Emergency Room (ER), hospital and outpatient settings. Resources include admission and discharge checklists, treatment algorithms, recommendations for forming a multidisciplinary team, patient education, and self-management materials, and key performance indicators to monitor whether standards of care are met or maintained, or should be improved. Conclusions: The HF Toolbox provides practical resources to simplify the management of patients with HF and to support the formation of HF programs in the Region. The Toolbox is aligned with current guideline recommendations and can support the management of patients from presentation in the ER, through hospital admission to outpatient care.

(c) 2019 The Author(s)

Published by S. Karger AG, Basel

\section{Introduction}

Heart failure (HF) is a complex clinical syndrome that places a significant burden on patients, caregivers, and healthcare systems [1]. Around 1-2\% of the population

\begin{tabular}{ll}
\hline KARGER & $\begin{array}{l}\text { @ } 2019 \text { The Author(s) } \\
\text { Published by S. Karger AG, Basel }\end{array}$ \\
E-Mail karger@karger.com & $\begin{array}{l}\text { This article is licensed under the Creative Commons Attribution- } \\
\text { NonCommercial-NoDerivatives 4.0 International License (CC BY- } \\
\text { NC-ND) (http://www.karger.com/Services/OpenAccessLicense). } \\
\text { Usage and distribution for commercial purposes as well as any dis- } \\
\text { tribution of modified material requires written permission. }\end{array}$
\end{tabular}

Prof. Samer Kabbani

Specialty Clinic Building

Maamari Street

Hamra, Beirut 1103 (Lebanon)

E-Mail kabbanisamer@ hotmail.com 
in developed countries has $\operatorname{HF}[2,3]$, and while there are limited data available on the prevalence and incidence of the condition within Asia-Pacific, Middle East and African countries (henceforth referred to as the "Region"), studies have reported that $0.5-6.7 \%$ of individuals in the Region have HF [4-9].

HF is associated with significant morbidity and mortality, and it greatly impacts patients' health-related quality of life [1]. The syndrome accounts for $0.65-1.53 \%$ of hospital admissions in the Region [6] with acute in-hospital mortality rates of $1-15 \%$ [6, 7, 10-14]. Following hospital discharge, mortality rates are high (30-day mortality rate $3.9-8.3 \%[10,15,16] ; 1$-year mortality rate 20 $28 \%[6,16,17] ; 5$-year mortality rate $50-68 \%[6,17])$, although they vary across the Region [18].

HF accounts for approximately $2 \%$ of healthcare expenditure worldwide, with up to $70 \%$ of the cost attributable to hospitalizations $[19,20]$. Overall healthcare expenditure on HF in Western countries and a number of countries within the Region is similar; however, Western countries typically spend more than developing countries on the primary treatment of HF, while developing countries tend to spend less on the initial management of patients and have greater costs in terms of readmissions and other indirect costs, compared with Western countries [19]. This highlights a potential opportunity for the Region to improve outpatient management and reduce hospitalization/re-hospitalization rates and associated costs. A registry of patients with $\mathrm{HF}$ in the Middle East revealed hospital readmission rates of 18 and $40 \%$ at 3 and 12 months, respectively [17]. This relatively high re-hospitalization rate may reflect a lack of adequate outpatient surveillance and management in specialized HF clinics, as well as underutilization of coronary intervention and device therapy [17].

Several differences in patient characteristics have been observed in the Region compared with Western countries. Patients diagnosed with HF in sub-Saharan Africa, the Middle East, China, and South/South-East Asia can be up to two decades younger than those in Western countries $[9,11,17]$. The etiology of HF is thought to differ in the Region compared with Western countries, with untreated congenital heart disease, rheumatic heart disease, cardiomyopathies, and hypertension as significant causes $[5,21]$. However, it should be noted that the etiology, prevalence, management, and outcomes of HF vary among the countries within the Region $[9,22]$. For instance, a large prospective cohort study of HF across Africa, Asia, the Middle East, and South America (International Congestive Heart Failure [INTER-CHF]) found that the primary etiology of HF in the Middle East and Asia was ischemic heart disease, as in Western countries $[10,23]$, while in Africa, the leading cause of HF was hypertension [23]. This finding is consistent with the subSaharan Africa Survey of Heart Failure (THESUS-HF), which found that nonischemic causes (most commonly hypertension) were the predominant etiology underlying $\mathrm{HF}$ in sub-Saharan Africa overall, with some variation across African countries [13].

Despite advances in the treatment of HF, morbidity and mortality remain high, and there are considerable unmet needs in the Region. These include a need to increase understanding of $\mathrm{HF}$, how to diagnose $\mathrm{HF}$ promptly, and the benefits of treatment among the public, healthcare workers, and policy makers. A lack of HF education for physicians, nurses, and other healthcare providers, as well as for patients and their families/caregivers, needs to be addressed. There is also a need to establish multidisciplinary teams (MDTs) for the management of patients with HF, and to identify quality measures to monitor performance and improve patient outcomes. The Region also suffers from limited resources for long-term patient follow-up, such as dedicated HF clinics and measures/protocols to prevent hospital readmissions.

An important factor in improving patient outcomes is implementation of practices that follow guideline recommendations. It is recognized that adherence to guidelines for the selection of appropriate medication class(es), titrated to target dosages, improves patient outcomes $[24,25]$. This may be important to address in the Region, as data from the recent QUALIFY study indicated that a relatively high proportion of patients in Asia and the Middle East, compared with Caucasian patients, were on treatment regimens with poor adherence to guideline recommendations [25]. In addition, practical measures such as establishing MDTs for the management of patients with HF, with structured discharge planning, provision of educational materials to encourage self-care and the set-up of outpatient HF management programs, along with the assessment of key performance indicators (KPIs), may improve patient outcomes [26, 27].

In this article, we propose a set of resources (a "HF Toolbox") to guide the management of patients with acute HF (AHF) and patients with chronic HF with reduced ejection fraction (HFrEF), in line with current recommendations, and to support the formation and enhancement of HF programs in the Region. The HF Toolbox will be useful for all healthcare professionals (HCPs) 


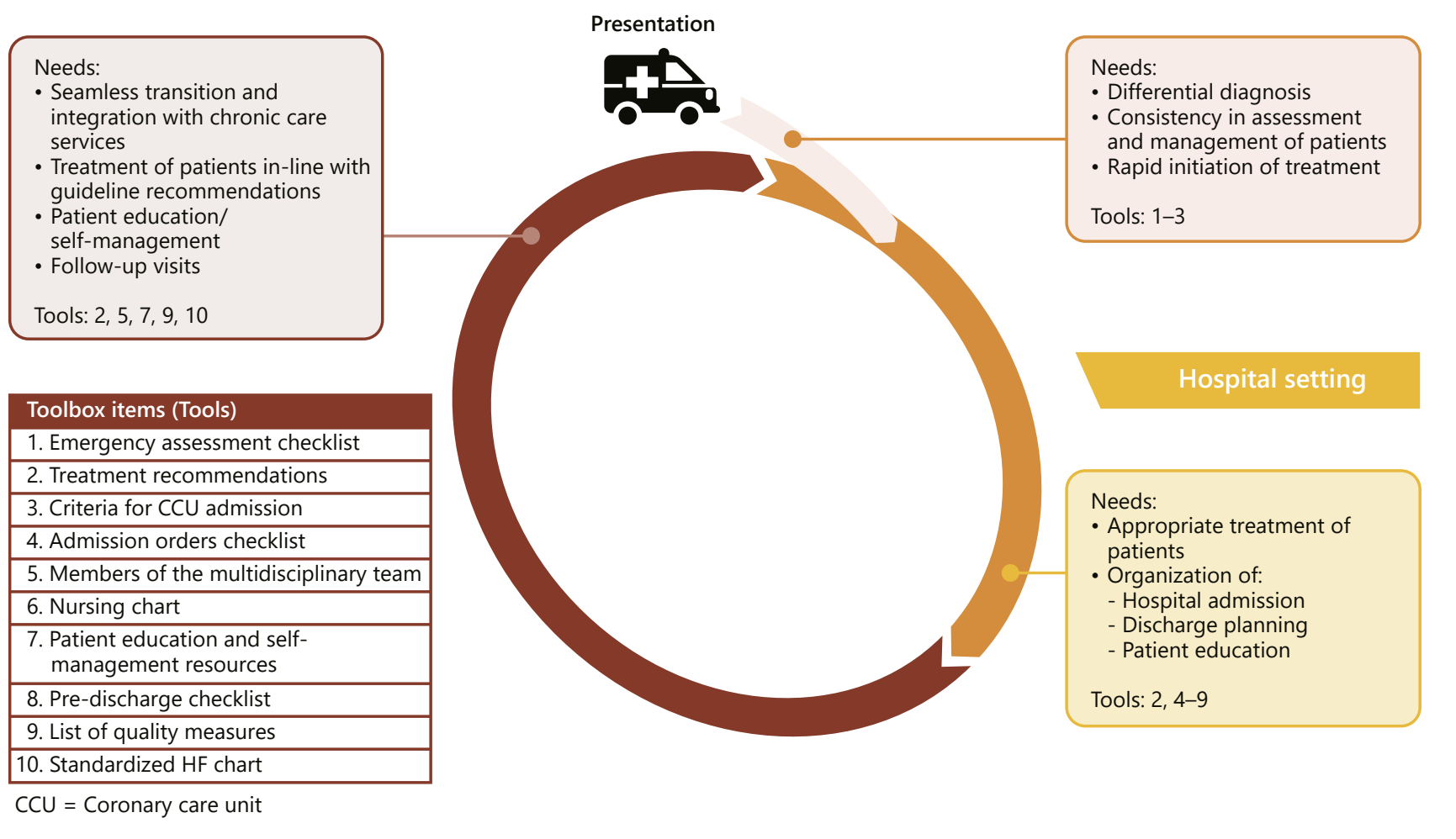

Fig. 1. An overview of the Heart Failure Toolbox.

who manage patients with AHF and patients with chronic HFrEF and may be particularly useful for centers that would like to have, or have already initiated, a set-up of a HF program. By facilitating the management of patients, the HF Toolbox will be valuable, particularly when the treating physician is not a cardiology specialist, as is often the case in the Region.

\section{Overview of the HF Toolbox}

We have developed a HF Toolbox to simplify the management of patients with HF from the presentation in the Emergency Room (ER), through hospital admission to outpatient care. The HF Toolbox is intended to guide the management of patients with AHF and patients with chronic HFrEF. As medical experts from across the Region with many years of experience in the management of patients with HF, we selected key resources for inclusion within the HF Toolbox that would help optimize the management of these patients (Fig. 1).

This HF Toolbox has been developed in line with the current guidelines for the management of HF, with the aim of demonstrating how to implement these standards in practice. The HF Toolbox comprises checklists, algorithms, and templates, as well as other practical resources. The resources included are not exhaustive but represent an approach to facilitate the management of patients with $\mathrm{HF}$, based on our experiences, current evidence, and international guidelines. Tools are intended to provide general guidance to support the management of patients, rather than specific, step-by-step guidance.

The selected tools provide a core resource for MDTs and include resources recommended by international professional societies, and items that we (the authors) have developed as a group. Given that the MDT is involved in addressing the needs of patients with HF 
throughout their journey (from presentation at the ER through to hospital admission and outpatient care), some resources will be of use in more than one setting.

The HF Toolbox is freely available to HCPs and tools can be used individually or in conjunction with one another and translated and adapted according to local needs. As such, the HF Toolbox is expected to be useful irrespective of healthcare infrastructure and available resources, which vary considerably across the Region. The HF Toolbox also includes a guide to allow users to easily identify resources of relevance.

\section{Items within the HF Toolbox}

\section{The ER Setting}

Within the Region, there is significant heterogeneity in AHF care, with ER teams rarely including HF specialists. As such, guidance in the form of checklists and algorithms should be used to provide consistency in the assessment and management of patients presenting with suspected AHF (including patients with de novo AHF and acute decompensation of chronic HF) and facilitate rapid initiation of treatment (Fig. 1).

Patients may present with wide-ranging symptoms, often complicated by the presence of comorbid conditions, making differential diagnosis challenging. We developed a two-part checklist for the emergency assessment of patients with suspected AHF (item 1) as a practical resource to guide history taking, physical examination, and initial investigations of patients who present at the ER with suspected AHF.

Initial management of patients with AHF is critical. The HF Toolbox includes a simplified algorithm (item 2a), developed based on our experiences and considering recommendations such as the European Society of Cardiology (ESC) guidelines for the diagnosis and treatment of acute and chronic HF [28]. This algorithm provides recommendations for the management of patients with AHF in the ER based on clinical features, including the presence of congestion and systolic blood pressure.

After patients have been stabilized in the ER, they may require admission to the Coronary Care Unit (CCU; or the Intensive Care Unit if not available), or to a regular ward for further treatment. HF Toolbox item 3 provides a list of criteria, based on the ESC guideline recommendations [28], to support physicians' decision making in terms of admission of patients to the CCU or to a regular ward. If any one of the criteria stated are met, the patient should be admitted to the CCU; if not, the patient can be admitted to a regular ward.

\section{The Hospital Setting}

In the hospital setting, appropriate treatment of patients with AHF is essential. In addition, co-ordination of hospital admission, discharge planning and patient education is vital, but may be poorly organized and implemented inconsistently [1]. We provide a number of resources, including checklists, charts, algorithms, a patient information and self-management booklet, and a list of KPIs, to optimize the in-hospital management of patients with AHF (Fig. 1).

We have produced an admission orders checklist (item 4), which we recommend completing for each patient upon hospital admission to support various aspects of their management in the inpatient setting. The checklist provides an opportunity for the admitting physician to establish a patient's clinical profile (according to the presence or absence of congestion and adequate perfusion or hypoperfusion) and create an individualized plan that clearly indicates what the patient requires in terms of pharmacological and nonpharmacological treatment strategies, assessments to be undertaken, monitoring by nursing staff and other nursing activities, and patient education.

The tools for use in the hospital setting include a treatment algorithm (item $2 \mathrm{~b}$ ) to guide the management of patients with AHF during the early phase of hospital admission. Like the simplified algorithm provided for use in the ER setting, this algorithm has been developed to facilitate the selection of appropriate treatment, in line with ESC guidelines [28]. However, this version (for use in the hospital setting) provides greater detail to determine an appropriate course of action based on the patient's clinical profile (determined by the presence or absence of congestion ["wet"/"dry" patients, respectively] and systolic blood pressure).

Successful management of patients with HF requires input from several HCPs with different skills and specialties, working together to form an MDT. We have developed a document (item 5) to provide recommendations for establishing an HF team, including identification of specialists and supporting staff who should be involved, and detailing the roles and responsibilities of each member of the team. Occupations are listed by priority (highest priority to lowest priority for inclusion within the team), so that institutions (particularly those with limited resources) can easily identify which roles are most vital in the multidisciplinary management of patients with HF. 
An HF specialist nurse is a core member of the MDT. Specialist nurses and members of the wider hospital nursing team may find a nursing chart, tailored to patients with HF, useful (item 6). This chart is designed to support the follow-up of patients with AHF during hospital admission, including detailed monitoring and assessment of the main organ systems. It also includes space to record details of the hospital admission and the patient's medical history, to ensure that all of the necessary information is documented on one chart, and space for an action list. This item has been adapted from a resource on a website that provides notes, printable reference sheets and study aids for nurses [29].

Educating patients on steps they can take to reduce their risk of future HF-related events and on the importance of self-care is key to improving long-term outcomes. We have produced a booklet that includes both patient information and self-management resources (item 7), which can be given to patients with AHF during their hospital stay and can be translated into the local language and adapted according to local needs and recommendations. This booklet provides a brief overview of various aspects of HF management and can be complemented by more detailed educational materials provided in the outpatient setting.

The booklet describes the symptoms that patients with HF may experience and provides recommendations in relation to nutrition and diet (e.g., maintaining a low sodium diet and managing fluid intake). The booklet also outlines medication options (including how to manage side effects that patients may encounter with different classes of HF medication), activity and rehabilitation (guidance on safely introducing a suitable exercise regime), weight management and other aspects of a healthy lifestyle. Advice on the importance of regular follow-up appointments is included, as well as when to seek further medical treatment and useful online educational resources and mobile applications. This information is complemented by the inclusion of a checklist, and links to other useful resources designed to help patients to monitor their condition and proactively manage their HF.

Within the booklet is a self-management checklist that we have developed for patients, to ensure they understand key aspects of self-management and identify areas where they may require additional education and advice from a nurse specialist. This is accompanied by descriptions of and links to several useful materials developed by the Heart Failure Association of the ESC [30]. Materials include a list of warning signs that should be discussed with a doctor or nurse, or that warrant calling for immediate help; diary pages to record symptoms and events; a chart to record signs including heart rate, blood pressure, and weight that patients can monitor themselves between outpatient appointments; and an appointment record to note the time and date of appointments with the healthcare team, and reminders of anything they might need to bring or wish to discuss at the appointment.

Prior to discharge from hospital, it is essential for the treating physician in the hospital to summarize information on the patient's status, history, and treatment plan to facilitate smooth transition from inpatient to outpatient care. We advise sharing patient records across the MDT, to ensure that all HCPs involved in follow-up care have access to the relevant information. The pre-discharge checklist (item 8) is adapted from the Heart Failure Clinical Pathway of the National Heart Centre Singapore. The checklist includes fields to record the etiology of HF, the patients' comorbidities, and the reason for decompensation, information on the treatment that has been initiated (which drug class[es] have been prescribed and which deemed unsuitable for the patient, with reasons, if applicable), whether left ventricular ejection fraction has been assessed, and whether the patient has been offered an implanted device (cardioverter defibrillator or cardiac resynchronization therapy defibrillator). The checklist also includes space to record information in relation to follow-up appointments and a list of discharge instructions to be followed and documented by the other members of the MDT; for example, HF education by nursing staff or instructions from a pharmacist regarding medication regime.

It is hoped that utilizing the simplified resources within the HF Toolbox will help ensure that HCPs adhere to guidelines for the treatment of HF. Assessment of measurable aspects of patient care, through the evaluation of KPIs, provides a means of quantifying adherence to guidelines and assessing the performance of the hospital/ healthcare organization in maintaining standards of care for HF. The HF Toolbox includes a list of quality measures, based on ESC guideline recommendations [28] (item 9a) that we recommend evaluating on a yearly basis. The KPIs in this list focus on the use of guideline-recommended therapies, patient and hospitalization outcomes, the provision of patient education, and patient assessment. 


\section{The Outpatient Setting}

The transition of patients from the hospital to the outpatient setting is an important stage in the management of patients with HF and should involve seamless integration with chronic care services [1]. The use of evidencebased therapies in line with guideline recommendations, the provision of patient education, and the organization of multiple follow-up visits are required to ensure that patient self-management and long-term care are optimized [1]. As a result, we provide various resources for the MDT, including a patient chart, a patient booklet, and a list of KPIs to monitor and support the long-term management of patients with HF (Fig. 1).

Adherence to guideline recommendations has been shown to improve outcomes for patients with HF [24, 25]. The HF Toolbox provides a summary, based on ESC guidelines [28], of recommendations for the pharmacological and nonpharmacological management of patients with chronic HFrEF in the outpatient setting (item $2 \mathrm{c})$. With reference to the relevant sections of the guidelines [28], this document includes information on the different classes of medications used in the treatment of HFrEF and situations in which they are recommended and highlights the importance of uptitration of medications to target dosages, since adequate dosing is a key factor in optimizing patient outcomes [24]. This document also summarizes recommendations for the use of implantable devices, the prevention of thromboembolism, and nonpharmacological interventions such as aerobic exercise.

The MDT described above (under The Hospital Setting) includes both hospital-based staff and auxiliary staff involved in outpatient care. The MDT document provided in the HF Toolbox for use in the hospital setting (item 5) is also provided for reference in the outpatient setting.

Regular follow-up visits are a fundamental aspect of outpatient care. It is useful if the assessments completed at these visits are standardized, so consistent data are collected at each visit to allow monitoring of patients' progress over the course of long-term follow-up. This may also aid data collection and help improve patient outcomes. The HF Toolbox includes a standardized patient chart (item 10) comprising record sheets to be completed at the first outpatient evaluation following hospital discharge (or first evaluation following referral from primary care, for patients with HFrEF who have not been hospitalized for acute decompensation) (sheets $1-3$ ), and at all subsequent follow-up visits (sheet 3). At the first outpatient visit, a detailed patient history should be taken, and the patient chart includes fields to capture basic information in relation to the patient, their caregiver(s) and their HF team, their medical history including non-HF problems, current HF management, HF diagnosis date, and left ventricular ejection fraction. Evaluations to be recorded at first and subsequent visits include vital signs, symptoms, New York Heart Association (NYHA) class, results from laboratory tests, and details of other assessments undertaken (e.g., electrocardiogram). Information on education, as provided by an HF nurse specialist, should also be captured at each outpatient visit.

As discussed above, patient education and self-management are important aspects of HF care. The patient information and self-management resources booklet provided for use in the hospital setting (item 7) can also be given to outpatients, if they have not been hospitalized, or did not receive it in hospital for any reason.

We have recommended monitoring of KPIs in the hospital setting; similarly, evaluation of KPIs in the outpatient setting can help ensure that standards of care are maintained. A list of quality measures relevant to the outpatient setting and aligned with ESC guideline recommendations is provided in the HF Toolbox (item 9b) [28]. Similar to the list of KPIs provided within the hospital setting resources, there is a focus on patient assessment, the provision of patient education, the use of guidelinerecommended therapies, and patient and hospitalization outcomes, and it is recommended that KPIs are measured on a yearly basis.

\section{Accompanying Guide}

The HF Toolbox also includes a guidance document for users, which provides an overview of the Toolbox and details of each of the items. This allows users to quickly and easily identify the resources that will be of most use to their institution(s). We recommend that items, such as completed checklists, are available to all members of MDTs, to provide all HCPs with a role in the management of patients with HF with as much information as possible, to allow smooth transitions between different care settings.

\section{Value of the HF Toolbox}

The Acute Cardiovascular Care Association (ACCA), Preventive Cardiovascular Nurses Association (PCNA), American Heart Association (AHA), Council on Cardiovascular Nursing and Allied Professions (CCNAP), and 
Table 1. Other resources available to support the management of patients with cardiovascular diseases

\begin{tabular}{|c|c|}
\hline Toolbox & Description \\
\hline $\begin{array}{l}\text { The CVD Prevention } \\
\text { Toolbox [31] }\end{array}$ & $\begin{array}{l}\text { Developed by the ESC, in collaboration with the EAPC } \\
\text { Consists of risk assessment and management tools for HCPs and policy makers } \\
\text { Resources include: } \\
\text { - Clinical practice guidelines } \\
\text { - Tools for decision-making, learning and risk assessment } \\
\text { - A health-related quality of life questionnaire (HeartQoL) } \\
\text { The tools, aside from the Expert Tool, are freely available }\end{array}$ \\
\hline $\begin{array}{l}\text { A Heart Healthy Toolbox: } \\
\text { Lifestyle Change Tools for } \\
\text { Health Care Professionals and } \\
\text { Their Patients [36] }\end{array}$ & $\begin{array}{l}\text { Developed by the PCNA } \\
\text { Includes the following to support heart healthy lifestyle education for adult patients in cardiology and primary care } \\
\text { settings: } \\
\text { - Professional resources } \\
\text { - Healthy eating resources } \\
\text { - Exercise and physical activity resources } \\
\text { - Total health resources } \\
\text { All tools are free to download } \\
\text { The PCNA has also developed five handouts for nurses and other HCPs to use with patients with HF [39] } \\
\text { - Topics include understanding HF, as well as treatment, exercise and diet } \\
\text { - An HF patient log is also provided, for patients to record information in relation to symptoms, weight and upcoming } \\
\text { appointments, along with any questions or concerns that they may have } \\
\text { - Handouts are available in paper and electronic formats and are free to download }\end{array}$ \\
\hline $\begin{array}{l}\text { CCNAP ESC guidelines } \\
\text { implementation toolkit [37] }\end{array}$ & $\begin{array}{l}\text { Developed by the CCNAP } \\
\text { Consists of freely available resources, such as videos and webcasts, to ensure that nurses and allied professionals in } \\
\text { Europe are trained to implement the latest ESC Guidelines }\end{array}$ \\
\hline $\begin{array}{l}\text { Global Heart Failure: An ACC } \\
\text { Education Program for the } \\
\text { Middle East, Africa and Asia } \\
\text { Regions [34] }\end{array}$ & $\begin{array}{l}\text { Developed by a group of HCPs from the Region, in collaboration with the ACC } \\
\text { Consists of disease education modules that cover both basic and advanced topics in relation to HF management and } \\
\text { include: } \\
\text { - Clinical cases } \\
\text { - Knowledge tests } \\
\text { Freely accessible for HCPs within the Region and all members of the ACC }\end{array}$ \\
\hline
\end{tabular}

ACC, American College of Cardiology; ACCA, Acute Cardiovascular Care Association; AHA, American Heart Association; AHF, acute heart failure; CCNAP, Council on Cardiovascular Nursing and Allied Professions; CVD, cardiovascular disease; EAPC, European Association of Preventive Cardiology; ESC, European Society of Cardiology; HCP, healthcare professional; HF, heart failure; PCNA, Preventive Cardiovascular Nurses Association; QoL, quality of life.

HF Toolbox for Healthcare Providers in Asia-Pacific, the Middle East, and Africa
Cardiology 2019;142(suppl 1):1-10

DOI: $10.1159 / 000496663$ 
American College of Cardiology (ACC) have developed resources to support the implementation of guideline recommendations and to guide the management of patients with HF (Table 1) [31-37]. However, it is our belief that this practical HF Toolbox will be a valuable resource for physicians, nurses and other HCPs in the Region for several reasons: the HF Toolbox is tailored to HCPs within the Region and the management of HF, while many of the currently available resources are aimed at HCPs in the United States of America or Europe and may not focus solely on the management of HF (an exception to this is the Global Heart Failure disease education program developed by HCPs from the Region, in collaboration with the ACC) [34]. Content is readily and freely available to any HCP interested in the improvement of HF care, is easy to follow, and is aligned with current guidelines for HF management. Selected, practical items from the guidelines have been simplified and included within the HF Toolbox, in a format that permits quick reference (e.g., simplified criteria for hospitalization). Institutions can pick which components of the HF Toolbox to use to support the management of patients with HF in their specific institution. They can also use the content of the HF Toolbox as a reference and adapt it according to their practices and the resources available. Checklists are available in both printable and editable electronic formats and print well in both color and black and white; users can select checklists in their preferred format for completion. Furthermore, the HF Toolbox includes a list of KPIs, so that HCPs can monitor the success of current and future practices.

The availability of this HF Toolbox in the Region may help support the implementation of practices that address key gaps and concerns in relation to HF management. Potential gaps in HF management in the Region include a lack of education for patients with $\mathrm{HF}$, a need to establish MDTs for the long-term management of patients with HF, a lack of quality measures to determine performance and improve patient outcomes, and limited resources for long-term patient follow-up. In addition, given the heterogeneity of current management practices in the Region, the HF Toolbox may assist in standardizing HF care. Reducing unmet needs and aligning management practices with guideline recommendations will likely result in improved patient outcomes $[26,38]$.

However, some limitations should be noted. While the resources included within the HF Toolbox have not been validated for use in the management of patients with HF, items have been adapted from materials used in clinical practice and developed based on international guideline recommendations. More objective evidence of the effectiveness of such resources is required, in terms of impact on patient outcomes. In addition, there may be limitations to the implementation of HF Toolbox recommendations, depending on the resources available within each country and within individual institutions.

\section{Conclusions}

In this article, we present a HF Toolbox that provides practical resources to guide the management of patients with HF (AHF and chronic HFrEF) and to support the formation of HF programs in Asia-Pacific, Middle East, and African countries (the Region). The HF Toolbox is aligned with current guidelines for the management of patients with HF and can be used to support the management of patients from presentation in the ER, through hospital admission to outpatient care. It is hoped that the dissemination of the HF Toolbox within the Region will guide the implementation of practices that help address key gaps and concerns in relation to HF management, and, ultimately, improve the outcomes of patients with HF in the Region.

\section{Interactive Files}

Interactive versions of the items are available as online supplementary material at www.karger.com/doi/10.1159/000496663.

\section{Acknowledgements}

- The authors would like to thank Dr. Feras Bader (United Arab Emirates), Dr. Annette Borromeo (Philippines), Prof. WeiChun Huang (Taiwan), Prof. Amam Chinyere Mbakwem (Nigeria), Dr. Anita Sharma (Australia), Prof. Chao-Hung Wang (Taiwan), and Prof. Jong-Chan Youn (South Korea) for their expert input regarding unmet needs in the region, and Miss Teo Lee Wah (Singapore) for her assistance with developing the predischarge checklist.

- The authors were assisted in the preparation of the manuscript by Hannah Birchby, a professional medical writer at CircleScience (Tytherington, UK), an Ashfield Company, part of UDG Healthcare plc. that was funded by Novartis Pharma AG, Basel, Switzerland. 


\section{Disclosure Statement}

- S.K. receives consultation honoraria from Novartis Pharma Services, Inc.

- W.A.H. has received speaking and consulting honorarium from Novartis, Servier, Bayer, MSD, Boehringer Ingelheim, and Pfizer.

- H.-B.L. has received personal fees from Novartis Corporation, Bayer, Medtronic, and Boston Scientific.

- J.C.M. has received speaker fees from Novartis, Boehringer Ingelheim, MSD, AstraZeneca, Janssen Pharmaceuticals, Lupin, Cipla, Abbott Healthcare, Dr. Reddy's Lab, and Bayer Pharmaceuticals. These companies have also provided honoraria for attending advisory board meetings in the last year.

- E.O. has received travel grants and speaker fees from Novartis, Servier, Merck Serono, Sanofi, and AstraZeneca.

- D.S. has received speaker honorarium and travel grants from Novartis, Servier, Pfizer, Boehringer Ingelheim, and Medtronic.
- N.T. has received speaker honorarium and travel grants from Novartis, Medtronic, Boston Scientific, Biotronik, and Amgen. $\mathrm{He}$ is on the medical advisory board for Novartis, Novo Nordisk, and Pfizer.

\section{Funding Sources}

Development of the Heart Failure Toolbox, writing/editorial support and open access fees were funded by Novartis Pharma AG, Basel, Switzerland. The sponsor reviewed the initial draft and subsequent versions of the manuscript for own data accuracy and for proprietary evaluation.

The authors received honoraria for their work on the Heart Failure Toolbox and their travel for associated face-to-face meetings was funded by Novartis Pharma AG, Basel, Switzerland.

\section{References}

1 Cowie MR, Anker SD, Cleland JG, Felker GM, Filippatos G, Jaarsma T, et al. Improving care for patients with acute heart failure: before, during and after hospitalization. ESC Heart Fail. 2014 Dec;1(2):110-45.

2 Mosterd A, Hoes AW. Clinical epidemiology of heart failure. Heart. 2007 Sep;93(9):113746.

3 Benjamin EJ, Blaha MJ, Chiuve SE, Cushman M, Das SR, Deo R, et al.; American Heart Association Statistics Committee and Stroke Statistics Subcommittee. Heart Disease and Stroke Statistics-2017 Update: A Report From the American Heart Association. Circulation. 2017 Mar;135(10):e146-603.

4 Report on cardiovascular disease in China (2011). Hu S, Kong L (ed). National Center for Cardiovascular Disease. Encyclopedia of China Publishing House: Beijing, China, 2014, pp 1-151. www.ecph.com.cn.

5 Pillai HS, Ganapathi S. Heart failure in South Asia. Curr Cardiol Rev. 2013 May;9(2):10211.

6 Ponikowski P, Anker SD, AlHabib KF, Cowie MR, Force TL, Hu S, et al. Heart failure: preventing disease and death worldwide. ESC Heart Fail. 2014 Sep;1(1):4-25.

7 Callender T, Woodward M, Roth G, Farzadfar F, Lemarie JC, Gicquel S, et al. Heart failure care in low- and middle-income countries: a systematic review and meta-analysis. PLoS Med. 2014 Aug;11(8):e1001699.

8 Shimokawa H, Miura M, Nochioka K, Sakata Y. Heart failure as a general pandemic in Asia. Eur J Heart Fail. 2015 Sep;17(9):884-92.

9 Sakata Y, Shimokawa H. Epidemiology of heart failure in Asia. Circ J. 2013;77(9):220917.

HF Toolbox for Healthcare Providers in Asia-Pacific, the Middle East, and Africa
10 Al-Shamiri MQ. Heart failure in the Middle East. Curr Cardiol Rev. 2013 May;9(2):174-8.

11 Kraus S, Ogunbanjo G, Sliwa K, Ntusi NA. Heart failure in sub-Saharan Africa: A clinical approach. S Afr Med J. 2016 Jan;106(1):2331.

12 Kengne AP, Dzudie A, Sobngwi E. Heart failure in sub-Saharan Africa: a literature review with emphasis on individuals with diabetes. Vasc Health Risk Manag. 2008;4(1):123-30.

13 Damasceno A, Mayosi BM, Sani M, Ogah OS, Mondo C, Ojji D, et al. The causes, treatment, and outcome of acute heart failure in $1006 \mathrm{Af}$ ricans from 9 countries. Arch Intern Med. 2012 Oct;172(18):1386-94.

14 Harikrishnan S, Sanjay G, Anees T, Viswanathan S, Vijayaraghavan G, Bahuleyan CG, et al.; Trivandrum Heart Failure Registry. Clinical presentation, management, in-hospital and 90-day outcomes of heart failure patients in Trivandrum, Kerala, India: the Trivandrum Heart Failure Registry. Eur J Heart Fail. 2015 Aug;17(8):794-800.

15 Guo Y, Lip GY, Banerjee A. Heart failure in East Asia. Curr Cardiol Rev. 2013 May;9(2): $112-22$.

16 AlHabib KF, Elasfar AA, Alfaleh H, Kashour T, Hersi A, AlBackr H, et al. Clinical features, management, and short- and long-term outcomes of patients with acute decompensated heart failure: phase I results of the HEARTS database. Eur J Heart Fail. 2014 Apr;16(4): 461-9.

17 Hassan M. Gulf CARE: Heart failure in the Middle East. Glob Cardiol Sci Pract. 2015 Oct; 2015(3):34.
18 Dokainish H, Teo K, Zhu J, Roy A, AlHabib KF, ElSayed A, et al.; INTER-CHF Investigators. Global mortality variations in patients with heart failure: results from the International Congestive Heart Failure (INTER$\mathrm{CHF}$ ) prospective cohort study. Lancet Glob Health. 2017 Jul;5(7):e665-72.

19 Cook C, Cole G, Asaria P, Jabbour R, Francis DP. The annual global economic burden of heart failure. Int J Cardiol. 2014 Feb;171(3): 368-76.

20 Dickstein K, Cohen-Solal A, Filippatos G, McMurray JJ, Ponikowski P, Poole-Wilson PA, et al.; ESC Committee for Practice Guidelines (CPG). ESC Guidelines for the diagnosis and treatment of acute and chronic heart failure 2008: the Task Force for the Diagnosis and Treatment of Acute and Chronic Heart Failure 2008 of the European Society of Cardiology. Developed in collaboration with the Heart Failure Association of the ESC (HFA) and endorsed by the European Society of Intensive Care Medicine (ESICM). Eur Heart J. 2008 Oct;29(19): 2388-442.

21 Cotter G, Cotter-Davison B, Ogah OS. The burden of heart failure in Africa. Eur J Heart Fail. 2013 Aug;15(8):829-31.

22 Lam CS, Teng TK, Tay WT, Anand I, Zhang S, Shimizu W, et al. Regional and ethnic differences among patients with heart failure in Asia: the Asian sudden cardiac death in heart failure registry. Eur Heart J. 2016 Nov;37(41): 3141-53.

23 Dokainish H, Teo K, Zhu J, Roy A, AlHabib KF, ElSayed A, et al.; INTER-CHF Investigators. Heart Failure in Africa, Asia, the Middle East and South America: the INTER-CHF study. Int J Cardiol. 2016 Feb;204:133-41. 
24 Poelzl G, Altenberger J, Pacher R, Ebner CH, Wieser M, Winter A, et al.; Austrian Working Group on Heart Failure. Dose matters! Optimisation of guideline adherence is associated with lower mortality in stable patients with chronic heart failure. Int J Cardiol. 2014 Jul; 175(1):83-9.

25 Komajda M, Cowie MR, Tavazzi L, Ponikowski P, Anker SD, Filippatos GS; QUALIFY Investigators. Physicians' guideline adherence is associated with better prognosis in outpatients with heart failure with reduced ejection fraction: the QUALIFY international registry. Eur J Heart Fail. 2017 Nov;19(11): 1414-23.

26 Riley JP, Masters J. Practical multidisciplinary approaches to heart failure management for improved patient outcome. Eur Heart J Suppl. 2016;18 suppl G:G43-52.

27 Iyngkaran P, Tinsley J, Smith D, Haste M, Nadarajan K, Ilton M, et al. Northern Territory Heart Failure Initiative-Clinical Audit (NTHFI-CA)-a prospective database on the quality of care and outcomes for acute decompensated heart failure admission in the Northern Territory: study design and rationale. BMJ Open. 2014 Jan;4(1):e004137.

28 Ponikowski P, Voors AA, Anker SD, Bueno $\mathrm{H}$, Cleland JG, Coats AJ, et al.; ESC Scientific Document Group. 2016 ESC Guidelines for the diagnosis and treatment of acute and chronic heart failure: the Task Force for the diagnosis and treatment of acute and chronic heart failure of the European Society of Cardiology (ESC)Developed with the special contribution of the Heart Failure Association (HFA) of the ESC. Eur Heart J. 2016 Jul; 37(27):2129-200.
29 Straight A. Nursing. Available from: http:// www.straightanursingstudent.com/studyaids/brain-sheets/.

30 The Heart Failure Association of the European Society of Cardiology. Heartfailurematters.org. Available from: http://www.heartfailurematters.org/en_GB.

31 The European Society of Cardiology. CVD Prevention Toolbox. Available from: https:// www.escardio.org/Education/PracticeTools/CVD-prevention-toolbox/CVD-Prevention-Toolbox.

32 Acute Cardiovascular Care Association of the European Society of Cardiology. ACCA Clinical Decision Making Toolkit. Available from: https://www.escardio.org/portal/site/Escardio/menuitem.c9f480f01a3ca18798f54de720 $2031 \mathrm{ca} /$ ?vgnextoid=f8b9463fd513b410VgnV CM1000004e03a8c0RCRD\&vgnextchannel= 0b3671c32f1f4510VgnVCM1000005303a8c0 RCRD\&vgnextfmt $=$ default\&vgnextlocale $=$ EN.

33 American Heart Association. Get With The Guidelines-HF Toolbox. Available from: http://www.heart.org/HEARTORG/Professional/GetWithTheGuidelines/GetWithTheGuidelines-HF/Get-With-The-GuidelinesHF-Toolbox_UCM_307815_Article.jsp\#. WWM6HYTyu70.

34 Global Heart Failure. An ACC Education Program for the Middle East, Africa, and Asia Regions. Available from: http://www.acc.org/ Heartfailurehub.
35 Acute Cardiovascular Care Association of the European Society of Cardiology. Clinical Decision Making Toolkit. Second edition. Available from: http://www.acc.org/Heartfailurehub.

36 Preventive Cardiovascular Nurses Association. The Heart Healthy Toolbox. Available from: http://pcna.net/clinical-tools/tools-forhealthcare-providers/heart-healthy-toolbox.

37 European Society of Cardiology. Council on Cardiovascular Nursing and Allied Professions (CCNAP) ESC guidelines implementation toolkit. Available from: https://www.escardio.org/Councils/Council-on-cardiovascular-nursing-and-allied-professions(CCNAP)/be-guidelines-smart.

38 Sanderson JE. Heart failure-a growing epidemic in Asia. Hong Kong Med J. 2004 Apr; 10(2):76.

39 Preventive Cardiovascular Nurses Association. Heart failure: educating your patients. Available from: http://pcna.net/clinicaltools/education-for-your-patients/heart-failure.

40 American Heart Association. TARGET:HF. Heart failure: medication optimization measures. Available from: http://www.heart.org/ idc/groups/heartpublic/@private/@wcm/@ hcm/@gwtg/documents/downloadable/ ucm_432945.pdf.

41 American Heart Association. TARGET:HF Heart failure discharge checklist. Available from: http://www.heart.org/idc/groups/ heartpublic/@private/@wcm/@hcm/@gwtg/ documents/downloadable/ucm_434627.pdf. 